\title{
Editorial
}

\section{From the Editor's Desk}

On behalf of Editorial Board Members, International Journal of Epilepsy, I am happy to present you Volume 3/2 issue of IJEP. This issue contains three interesting original articles. The article by $\mathrm{Dr}$. Gautam Das and colleagues highlights the cognitive outcomes in epilepsy patients. Their study shows that epileptic patients show impairment in working memory function, visuo-spatial skills, processing speed, visuo-perceptual attainment and reasoning ability. The deficit was more pervasive in patients of GTCS. It also shows that patients with GTCS and CPS exhibit lower cognitive scores than the healthy controls.

Dr. Puja Kumari, Dr. Lekha Saha and Dr. Neha Rana in a novel study from Post Graduate Institute of Medical Education \& Research (PGIMER), Chandigarh assessed anti-kindling effect of allopregnanolone alone and its interaction with sodium valporate in pentylenetetrazole induced kindling model. The study revealed that the combination of low dose of allopregnanolone with low dose of sodium valproate showed a similar beneficial effect to that of a higher dose of sodium valproate in significantly reducing the number of kindled animals as compare to pentylenetetrazole control group. The study has opened fresh avenues for further research in this direction.

Akriti Gupta et al. from Ludhiana studied the felt stigma associated with epilepsy during arranged marriages that affects women more frequently and intensely. South Asian WWE experience more felt stigma than men before and after arranged marriages and this might impact a number of health related psychosocial outcomes.
Other highlights of this issue are special review articles on maternal and neonatal complications during pregnancy in women with epilepsy, a monograph on novel anti-epileptic drug perampanel and drug repositioning. Rapid advances in pharmacotherapy and bioinformatics has led to the discovery and growing popularity of drug repositioning which includes re-investigating or recycling of existing drugs for new indications. The latter concept is again upcoming and new to the field.

To make this issue more interesting for its readers, two case reports and an 'image of the month' have been incorporated.

We lost great personality and one of doyen in the field of epilepsy Prof. Uwe Heinemann on August 31, 2016. This journal contains the obituary of this great personality who contributed in the field of epilepsy.

We hope that you will find all the articles useful. You will also find other feature in this issue like teaching EEGs, MCQs on epilepsy surgery interesting to read.

Your valuable suggestions/inputs are solicited.

Wish you all a very happy New Year 2017.

Best wishes and kind regards,
Man Mohan Mehndiratta Editor-in-Chief 